\title{
Definitive Measurements of a Supermassive Black Hole and Its Surrounding Mass
}

\author{
Linda Dressel \\ Space Telescope Science Institute, Baltimore, MD, USA
}

\begin{abstract}
A discrete thin rotating emission-line disk is ideal for determining the mass of a supermassive black hole in the nucleus of a galaxy. One can use high resolution imaging in combination with a matrix of spatially resolved velocity measurements to confirm the simple geometry and kinematics of the disk. If the disk remains bright and orderly beyond the sphere of influence of the black hole, with motion driven predominantly by gravitational force, one can also measure the density of the surrounding matter in the nucleus. This is being done for the bright Liner galaxy NGC 3998.
\end{abstract}

\section{Introduction}

The central structure of a galaxy holds clues to its formation and evolution. Knowledge of the present structure is needed to test theories about how the spheroidal component forms, how a supermassive black hole grows (and stops growing), how the black hole affects the distribution of matter surrounding it, and how merging systems with black holes evolve. HST observers have made several break-throughs in determining the central structure of the spheroidal component of galaxies. They have shown that spheroids have a central light cusp (Ferrarase et al. 1994, Faber et al. 1997). Modelling of the observed powerlaw surface brightness profiles has shown that light density increases inward to the limiting resolution, even when the innermost surface brightness profile has the appearance of a core (Gebhardt et al. 1996). A growing body of stellar and gas kinematic observations has shown that galaxies have a supermassive black hole in the nucleus whose mass depends on the gravitational potential of the spheroidal component, as measured by its luminosity or velocity dispersion (Ferrarrese et al. 2000, Gebhardt et al. 2000). As seen in M31, the black hole can be offset from both the physical center and the systemic velocity of the spheroid (Kormendy \& Bender 1999). A coherent scenario is forming, in which adiabatically growing black holes in undisturbed galaxies cause a cusp to form and cause the center of the spheroid to become more circular and axisymmetric, which inhibits further growth of the black hole. Subsequent mergers produce triaxial shapes and binary black holes, which deplete the cusp by scattering stars as they wander and proceed towards coalescence (Merritt 2000). Accurate measurements of the density profiles and the mass-to-light (M/L) profiles in spheroids, and of the exact location of the black hole, will help us refine these theories. 
We have been making increasingly accurate measurements of black holes and of the density profile of the surrounding matter. Mass modelling from stellar kinematics is evolving from self-consistent 2-integral models to 3-integral models with anisotropic velocity dispersions (Cretton \& van den Bosch 1999, Gebhardt et al. 2000). Some basic problems for stellar modelling are that velocity dispersion is always a significant component relative to rotation velocity, dispersion can be observed only in one dimension but may be anisotropic, line profiles are viewed in summation along the line of sight through the entire galaxy, and one must either assume or model M/L. The HST observation of M87 by Ford et al. (1994) revealed the possibility of using nuclear emission line disks to measure mass more directly. Rotating gaseous disks are geometrically and kinematically simpler than stellar spheroids, but one must find unobscured disks with high surface brightness, with well ordered rotation (undisrupted by any activity that may power the unusual brightness), and with streaming velocity dominant over velocity dispersion (which can be difficult to interpret). To find and measure such disks, I have used the Hubble Space Telescope to observe some of the nearest brightest Liner nuclei. Their host galaxies were initially selected as powerful compact radio sources (Condon \& Dressel 1978), then were found or confirmed to be unusually bright Liners (O'Connell \& Dressel 1978). They therefore form a comparison sample of active nuclei to be contrasted to the normal galaxies that dominate stellar absorption line programs.

\section{Data and Measurements}

I first imaged the nuclei of 12 galaxies in $\mathrm{H} \alpha+[\mathrm{NII}]$ emission and in one or two adjacent continuum bands with WFPC2. The continuum-subtracted emission line images show that each nucleus has a bright sub-arcsecond disk-like formation of ionized gas. I subsequently observed 8 of the nuclei with STIS in the spectral region containing $\mathrm{H} \alpha+[\mathrm{NII}] \lambda 6548,6584$ and $[\mathrm{SII}] \lambda 6717,6731$. The 0.1 arcsec slit was placed along the major axis of the emission line disk and in 2 or 4 parallel positions to map the emission-line kinematics. Here I present results for NGC 3998 , which was observed at 5 positions.

NGC 3998 has one of the brightest nuclear gas disks in the nearby universe. It is several times brighter than the nuclear disk in M87. It is also one of the nearest bright Liners. At a distance of $23 \mathrm{Mpc}$, it has a linear scale of $5.6 \mathrm{pc}$ per $0.05^{\prime \prime}$ (per PC or STIS pixel). The ratio of F547M and F791W PC images shows that reddening throughout the nuclear region of this $\mathrm{S} 0$ galaxy is exceptionally low. The ionized disk is compact and relatively featureless. The disk is shown in Figure 1a, with the STIS slit in the central observing position superposed.

Each row of pixels in a rectified STIS spectral image gives a spectrum from an increment of 0.05 arcsec along the 0.1 arcsec slit. For each row, I fit a linear baseline to the continuum and Lorentzian profiles (which fit better than gaussians) to the emission lines. $\mathrm{H} \alpha$ was fit independently, the [NII] $\lambda 6548,6584$ line fits were constrained to each other in wavelength ratio, width, and intensity ratio, and the $[\mathrm{SII}] \lambda 6717,6731$ line fits were constrained to each other in wavelength ratio and width. Nearest the nucleus, a broad blue wing to the $\mathrm{H} \alpha$ line was fit by an additional Lorentzian profile. The consistency of the 3 velocity 

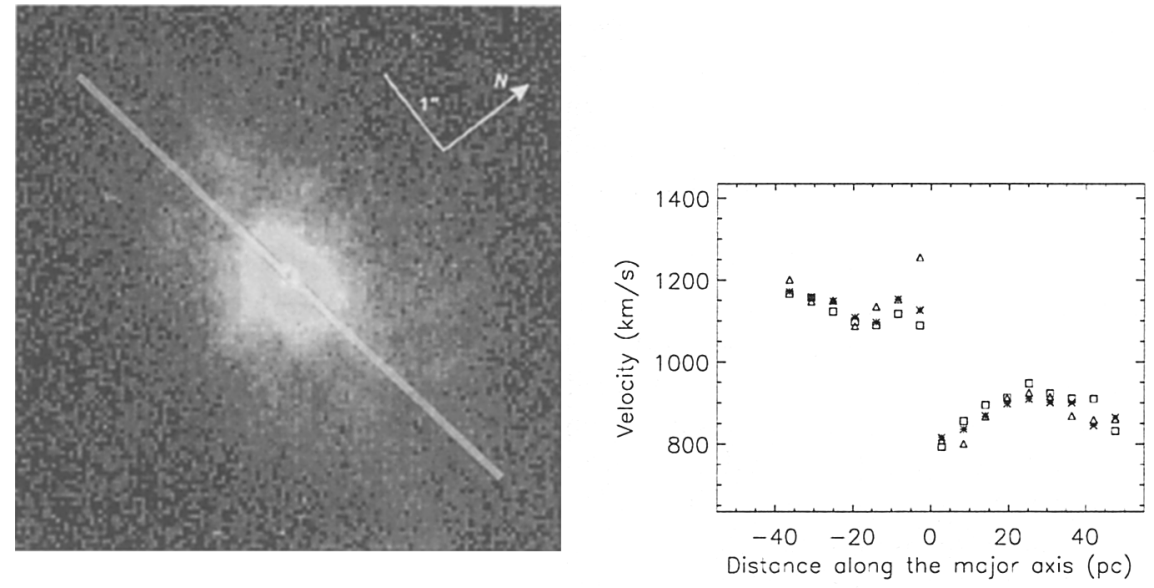

Figure 1. a) $\mathrm{H} \alpha+[\mathrm{NII}]$ image with centrally placed STIS slit. b) Major axis rotation curve with velocities determined from $\mathrm{H} \alpha$ (triangle), [NII] $(*)$, and [SII] (square).

centroids of the main $\mathrm{H} \alpha$ component, the [NII] pair, and the [SII] pair gives a measure of the error in the velocity determination for each row.

\section{Modelling}

The major axis rotation curve (Figure 1b) clearly shows the influence of a black hole in its inner portion, then rolls over as the distributed mass begins to dominate the gravitational potential. The following caveats apply to the accuracy of the measured velocities. Smearing by the point spread function of the telescope is most consequential for the two points nearest the kinematic center, which would show even greater rotational velocities if less blended. The [NII] velocity at the innermost receding point is poorly determined because of blending with the $\mathrm{H} \alpha$ line. Several of the inner [SII] points have relatively low rotational velocities, especially on the receding curve. The intensity ratio of the [SII] lines for these points shows that their associated critical densities have been exceeded, indicating that higher rotational velocities are increasingly depleted from the line profile as one approaches the denser inner regions. The problematic [NII] and [SII] measurements will not be used in the modelling.

The major axis rotation curve is consistent with a black hole embedded in a nearly uniform density. As expected for a rotating disk, the observed velocity is close to systemic at the points on the minor axis. The isophotes in the $\mathrm{PC}$ emission line image imply that the disk is at an inclination of about $40^{\circ}$, which will be assumed in the models. A trial fit in figure $2 \mathrm{a}$, for a $1 \times 10^{8}$ $\mathrm{M} \odot$ black hole embedded in a uniform density of $1600 \mathrm{M} \odot / \mathrm{pc}^{3}$, shows that the data points are not distributed symmetrically. In particular, the inner points on the receding side have a lower rotation velocity where the gas has a higher velocity dispersion. This may indicate that the random motions of gas clouds are contributing to the support of the disk against gravity. It is not possible 

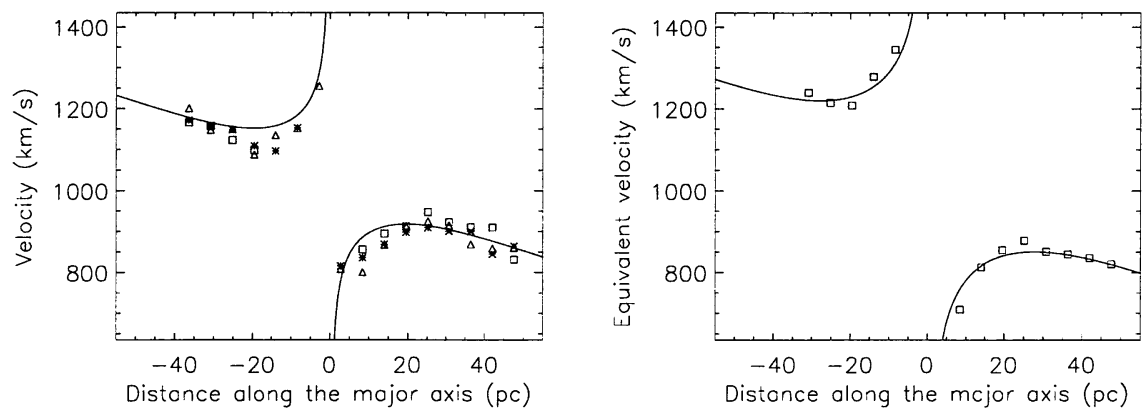

Figure 2. a) Model fit to major axis rotation curve: $1 \times 10^{8} \mathrm{M} \odot+$ $1600 \mathrm{M} \odot / \mathrm{pc}^{3}$. b) Model fit to major axis rotation curve (with $\mathrm{H}$ alpha, [NII], [SII] velocities averaged) adjusted to include velocity dispersion: $3.5 \times 10^{8} \mathrm{M} \odot+2000 \mathrm{M} \odot / \mathrm{pc}^{3}$.

to determine from these observations the exact nature of the non-rotational velocities, but most schemes for taking them into account involve either assuming that they play no role ("turbulence") or that the rms velocity adds quadratically to the rotation velocity with a scaling factor determined by the model. If one adds the average random and rotational energies, and assumes that the velocity dispersion is isotropic, the scaling factor for the observed mean squared velocity is 3 . The "equivalent" rotation velocity resulting from this treatment of the velocity dispersion, projected to inclination $40^{\circ}$, is shown in Figure $2 \mathrm{~b}$. A trial fit is shown for a $3.5 \times 10^{8} \mathrm{M} \odot$ black hole embedded in a uniform density of $2000 \mathrm{M} \odot / \mathrm{pc}^{3}$. The best estimates for the central mass and mass density from this first order modelling therefore lie between 1 and $3.5 \times 10^{8} \mathrm{M} \odot$ and 1600 and $2000 \mathrm{M} \odot / \mathrm{pc}^{3}$.

Acknowledgments. This work was supported by grants HST-GO-5924, HST-GO-7354, and NASA LTSA grant NAG5-8375.

\section{References}

Condon, J. J. \& Dressel, L. L. 1978, ApJ, 221, 456

Cretton, N. \& van den Bosch, F.C. 1999, ApJ, 514, 704

Faber, S. M., Tremaine, S., Ajhar, E. A. et al. 1997, AJ, 114, 1771

Ferrarese, L. \& Merritt, D. 2000, ApJ, 539, L9

Ferrarese, L., van den Bosch, F. C., Ford, H. C., et al. 1994, AJ, 108, 1598

Ford, H. C., Harms, R. J., Tsvetanov, Z. I., et al. 1994, ApJ, 435, L27

Gebhardt, K. Bender, R., Bower, et al. 2000, ApJ, 539, L13

Gebhardt, K., Richstone, D., Kormendy, J., et al. 2000, AJ, 119, 1157

Gebhardt, K., Richstone, D., Ajhar, E. A. et al. 1996, AJ, 112, 105

Kormendy, J. \& Bender, R. 1999, ApJ, 522, 772

Merritt, D. 2000, in ASP Conf. Ser. Vol. 197, 221

O'Connell, R. W. \& Dressel, L. L. 1978, Nature, 276, 374 